\title{
AVALIAÇÃO DO ESTADO NUTRICIONAL DE PACIENTES ADULTOS INTERNADOS EM UMA FUNDAÇÃO HOSPITALAR DA CIDADE DE CASCAVEL - PR
}

\section{EVALUATION OF THE NUTRITIONAL STATE OF ADULT PATIENTS INTO A HOSPITAL FOUNDATION OF THE CITY OF CASCAVEL - PR}

\author{
Marianela Díaz Urrutia ${ }^{1}$, Marcia Machado ${ }^{1}$, Marina Horst ${ }^{1}$, Mirely Sharlau ${ }^{1}$, Rafael \\ Dewes Lenz ${ }^{1}$, Natalia Morando1, Débora Regina Hendges Pappen²
}

${ }^{1}$ Nutricionista, Centro Universitário Fundação Assis Gurgacz (FAG). ${ }^{2}$ Nutricionista Mestre, Docente do
Curso de Nutrição, Centro Universitário Fundação Assis Gurgacz (FAG).
*Autor correspondente: marianeladiazurrutia@gmail.com, https://orcid.org/0000-0001-5047-953X

DOI: $10.35984 /$ fjh.v2i1.154

\section{RESUMO}

O monitoramento constante do estado nutricional de pacientes hospitalizados, mediante uso de ferramentas de avaliação, tais como a determinação do Índice de Massa Corporal (IMC) é fundamental para o rastreamento de indivíduos em risco nutricional, pois este fato pode refletir em complicações clínicas que poderiam ser evitadas com um tratamento nutricional adequado. O presente trabalho objetivou avaliar o IMC de pacientes adultos hospitalizados em uma fundação hospitalar da cidade de Cascavel - PR. Foi aplicada triagem nutricional em 189 pacientes, onde foi mensurado o peso e a altura através de avaliação antropométrica, para logo serem classificados de acordo com o IMC. Houve diferença significativa $(p=0,0077)$ entre os grupos classificados segundo o estado nutricional pelo IMC, onde o grupo de pacientes com magreza foi significativamente menor quando comparado aos demais grupos (eutrofia, sobrepeso e obesidade) que não diferiram estatisticamente $(p>0,05)$ entre si. Sendo que no grupo de magreza a maior porção de pacientes correspondia a idosos com idade superior a 70 anos, evidenciando uma vulnerabilidade nutricional na referida faixa etária. 0 IMC, como ferramenta avaliativa isolada, carece de exatidão para estabelecer conclusivamente o risco nutricional de pacientes hospitalizados. Sendo necessário, conjugar outras informações para determinar o risco de desnutrição real de pacientes internados em hospitais.

Palavras chave: estado nutricional, hospitalizados, índice de massa corporal

\begin{abstract}
Constant monitoring of the nutritional condition of hospitalized patients through the use of assessment tools, such as the determination of Body Mass Index (BMI), is essential for the tracking of individuals at nutritional risk, as this fact may reflect on clinical complications that could be avoided with proper nutritional treatment. This study aimed to evaluate the BMI of hospitalized adult patients in a hospital foundation in the city of Cascavel - PR. Nutritional screening was applied to 189 patients, where weight and height were measured by anthropometric assessment, to be classified according to BMI. There was a significant difference ( $p=0.0077)$ between the groups classified according to nutritional conditions by $B M I$, where the group of patients with thinness was significantly smaller when compared to the other groups (eutrophy, overweight and obesity) that did not differ statistically $(p>0.05)$ to each other. In the thinness group, the largest portion of patients corresponded to elderly over 70 years old, showing a nutritional vulnerability in this age group. BMI, as an isolated assessment tool, lacks accuracy to conclusively establish the nutritional risk of hospitalized patients. It is necessary, to combine other information to determine the risk of desnutrition in hospital patients.
\end{abstract}

Key words: nutritional status, hospitalized, body mass index 


\section{INTRODUÇÃO}

A avaliação e monitoramento nutricional de pacientes hospitalizados deve ser constante, mediante uso de diversas ferramentas, a fim de estabelecer o melhor tratamento alimentar e nutricional para os mesmos e assim, reduzir o tempo de internamento que se reflete em menores custos por possíveis complicações clínicas (DUCHINI et al., 2010).

Por outro lado, a realização de uma triagem nutricional de pacientes hospitalizados, tem o intuito de verificar se o indivíduo precisa de uma avaliação mais profunda (MAHAN e RAYMOND, 2018). Sendo que no Brasil, o Ministério da Saúde, estabelece a aplicação obrigatória de protocolos para triagem e avaliação nutricional de pacientes internados pelo SUS (BRASIL, 1998).

Com base nos resultados de uma triagem, é essencial o acompanhamento desses pacientes, os quais devem ser priorizados, principalmente, quando em situação de risco nutricional com o objetivo de verificar e investigar as alterações nutricionais, e optar pela melhor intervenção que vise a adequação das necessidades de aporte energético e nutricional para a recuperação da saúde do indivíduo (BOSCO, et al., 2013).

Para tanto, se faz necessário um critério de padronização antropométrica com estimativas que assegurem o máximo de eficiência em relação aos valores de composição corporal, massa magra e tecido adiposo, de modo que o hospital estabeleça essas técnicas de avaliação do estado nutricional e sistematize sua aplicação, pois estas detectam o risco de desnutrição hospitalar e auxiliam no tratamento de melhora do prognóstico do paciente (FIDELIX, et al., 2013).

O Índice de Massa Corporal (IMC), é obtido a partir da relação entre o peso e a altura do indivíduo avaliado, para logo ser classificado conforme o estado nutricional que varia, para adultos entre: graus de magreza, eutrofia, sobrepeso e graus de obesidade, e para idosos varia entre: magreza, eutrofia e sobrepeso (MAHAN, RAYMOND, 2018).

Tendo em vista, que pacientes acamados e com impossibilidade de se locomover, demandam a utilização de instrumentos de alta tecnologia que demandam altos custos às instituições hospitalares, fazendo inviável o uso das mesmas para conferir dados como peso e altura com exatidão (DIAS, HORIE e WAITZBERG, 2006).

Dessa maneira, estes dados podem ser estimados mediante uso de fórmulas, que requerem medidas de determinadas porções do corpo, tais como, altura do joelho, circunferências de braço e panturrilha e dobras cutâneas (MITCHELL e LIPSHITZ, 1982).

O presente trabalho teve como objetivo analisar o estado nutricional de pacientes maiores de 18 anos internados em uma fundação hospitalar da cidade de Cascavel - PR, mediante a aferição ou estimativa do peso e altura dos mesmos, e concomitantemente, discutir a efetividade desta ferramenta para a identificação de pacientes com necessidades nutricionais especiais.

\section{METODOLOGIA}

O presente trabalho trata-se de um estudo descritivo, em que, foram coletados dados antropométricos adquiridos diretamente de 189 pacientes avaliados durante 0 
período de Estágio Supervisionado realizado em uma Fundação Hospitalar da cidade de Cascavel, Paraná.

Os pacientes avaliados foram adultos, maiores de 18 anos, de ambos os sexos, internados no período de 30 de julho de 2019 e 03 de setembro do mesmo ano, internados em todos os setores do estabelecimento (Unidade de Terapia Intensiva (UTI), Alas e Pisos).

Durante as avaliações antropométricas, foi determinada a altura e o peso corporal dos indivíduos, mediante dois procedimentos, que dependiam do estado de consciência do paciente. Por um lado, quando o paciente estava consciente e em condições de se levantar da cama, ambos dados eram aferidos em uma balança digital antropométrica do estabelecimento. Por outro lado, quando o paciente estava inconsciente ou consciente, porém impossibilitado de sair da cama, estes dados eram estimados mediante uso de fórmulas, sendo elas, segundo Chumlea et al., (1985):

- Estimativa de peso pela circunferência de panturrilha:

Homens: $(0,98 \times \mathrm{CP})+(1,16 \times \mathrm{AJ})+(1,73 \times \mathrm{CB})+(0,37 \times \mathrm{PSE})-81,69$

Mulheres: $(1,27 \times \mathrm{CP})+(0,87 \times \mathrm{AJ})+(0,98 \times \mathrm{CB})+(0,40 \times \mathrm{PSE})-62,35$

- Estimativa de altura pela altura do joelho:

Homem: $64,19-(0,04 \times \mathrm{I})+(2,02 \times \mathrm{AJ})$

Mulher: $84,88-(0,24 \times \mathrm{I})+(1,83 \times \mathrm{AJ})$

$\mathrm{CP}$ : circunferência da panturrilha $(\mathrm{cm})$; CB: circunferência do braço $(\mathrm{cm})$ AJ: Altura dos joelhos (cm); PSE: prega subescapular $(\mathrm{mm})$.

Sendo que, os dados das circunferências de braço e panturrilha, assim como também, a altura do joelho, foram determinadas mediante uso de fita métrica e a prega subescapular, foi determinada pela utilização de adipômetro científico.

A classificação do IMC foi de acordo com o preconizado pela OMS (1995; 1997), sendo este considerado de 18,5 a $24,9 \mathrm{~kg} / \mathrm{m}^{2}$ eutrofia, $<18,5 \mathrm{~kg} / \mathrm{m}^{2}$ magreza, $>24,9 \mathrm{~kg} / \mathrm{m}^{2}$ sobrepeso e > de $29,9 \mathrm{~kg} / \mathrm{m}^{2}$ obesidade para adultos. Já para a os pacientes acima de 65 anos, o IMC foi classificado de acordo com o proposto por Lipschitz (LIPSCHITZ, 1994), sendo $<22 \mathrm{~kg} / \mathrm{m}^{2}$ magreza, de 22 a $27 \mathrm{~kg} / \mathrm{m}^{2}$ eutrofia e $>27 \mathrm{~kg} / \mathrm{m}^{2}$ excesso de peso.

Depois do IMC ser determinado para cada paciente avaliado, os indivíduos foram distribuídos de acordo com a faixa etária em quatro grupos, onde: Grupo 1: idade entre 18 - 30 anos; Grupo 2: idade entre 31 - 50 anos; Grupo 3: idade entre 51 - 70 anos; e Grupo 4: idade > 70 anos.

Todos os dados foram avaliados mediante análise de variância (ANOVA) no programa Microsoft Excel 2010 e as médias foram testadas pelo teste de Tukey considerando um nível de significância de 5\%.

\section{RESULTADOS E DISCUSSÃO}

Na Figura 01, observa-se o percentual de pacientes avaliados de acordo com o estado nutricional determinado pelo IMC. Onde é possível verificar que houve diferença estatística entre os grupos ( $p=0,0077)$, em que o percentual de pacientes com magreza foi estatisticamente menor que os grupos de pacientes eutróficos, com sobrepeso e obesos, que por sua vez, não diferiram estatisticamente entre si.

Em estudo de Franco e Morsoletto (2011), foram apontados inúmeros fatores tais como fisiológicos, patológicos, etários ou psicológicos como importantes influências no estado nutricional de pacientes hospitalizados, onde se destacou o prognóstico e o grau de injúria do paciente na contribuição seja da estagnação ou do 
agravamento do quadro de nutrição deste paciente, o que pode justificar o resultado apresentado na figura 1 do presente estudo, pois neste caso não houve uma seleção de diagnósticos, visto que todos os pacientes internados no hospital são triados e no ato desta triagem, alguns se encontram internados somente para procedimentos eletivos e não apresentam maiores complicações que venham a comprometer seu estado catabólico na vigência do internamento.

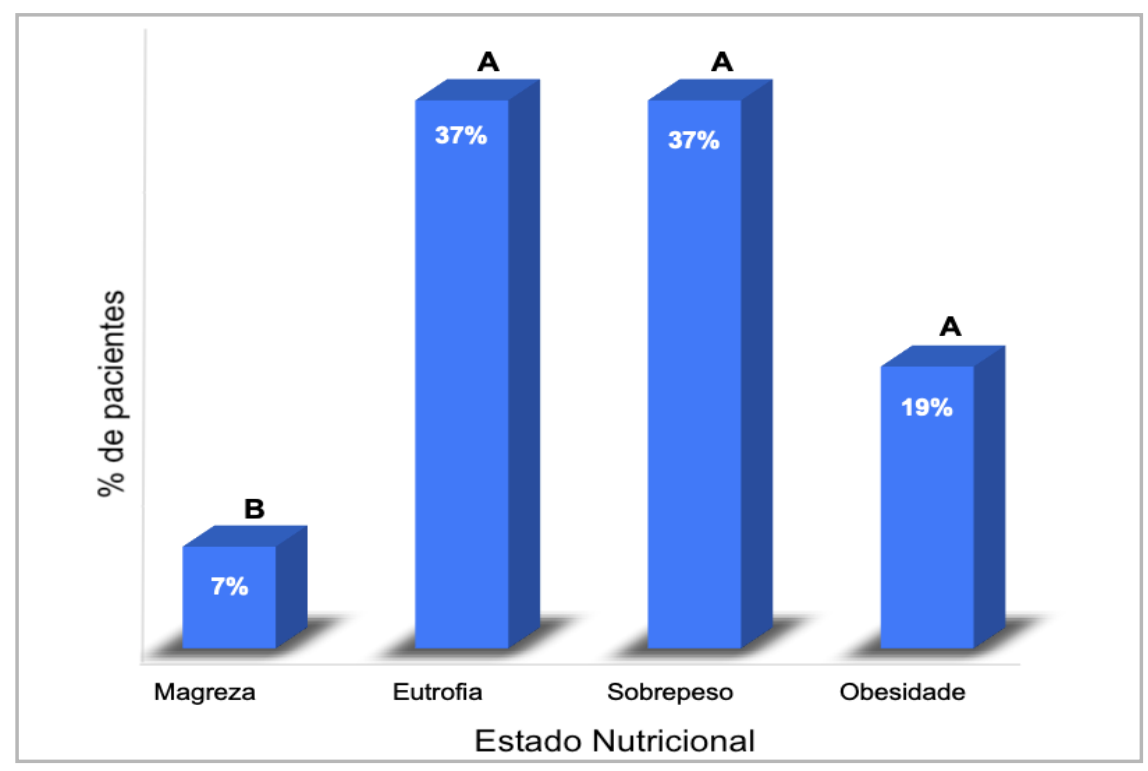

Figura 01. Percentual de pacientes avaliados classificados pelo estado nutricional.

*Os tratamentos com letras diferentes diferiram estatisticamente no teste de Tukey $(p<0,05)$.

Em seguida, na Figura 02, observa-se o percentual de pacientes por estado nutricional distribuídos de acordo com a faixa etária.

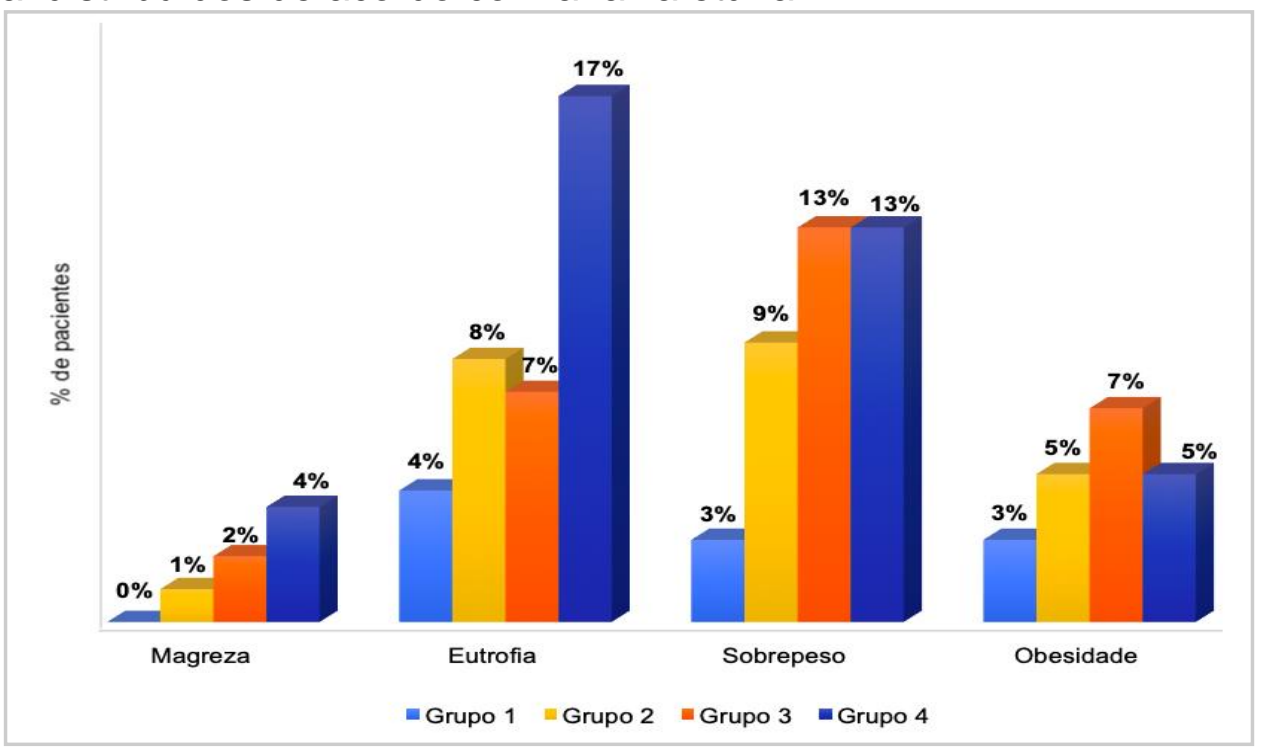

Figura 02. Estado nutricional por faixas etárias de pacientes adultos maiores de 18 anos, hospitalizados em uma fundação hospitalar na cidade de Cascavel - PR

Grupo 1: Pacientes com 18 - 30 anos; Grupo 2: Pacientes com 31 - 50 anos; Grupo 3: Pacientes com 51 - 70 anos; Grupo 4: Pacientes maiores de 70 anos.

Observando a Figura 02, é possível conferir que, do total pacientes avaliados, a menor parte encontrava-se em estado nutricional de magreza, sendo que dentro 
deste grupo a maioria dos indivíduos apresentava idade superior a 70 anos. Quanto à porção de indivíduos eutróficos, 17\% do total avaliado, tinham idade acima de 70 anos. Em relação ao sobrepeso, metade dos indivíduos pertenciam à faixa etária superior a 50 anos. Por outro lado, dos pacientes obesos, $7 \%$ do total apresentava idade entre 51 e 70 anos.

Analisando os resultados apresentados na Figura 02 em relação à prevalência do estado de magreza observado no determinado grupo, este deve ser considerado significativamente devido ao ciclo de vida destes pacientes, apontado em estudo de Paula, et al., (2016) que relaciona o envelhecimento com a imposição de modificações na composição corporal dos idosos, caracterizado por sinais sarcopênicos, onde ocorre a perda progressiva de massa e força muscular. Por outro lado, Fernandes e Bezerra (2006), evidenciam em seu estudo com pacientes idosos portadores de Doença Pulmonar Obstrutiva Crônica (DPOC), que esta condição nutricional deste mesmo grupo, pode ser relacionada com o fator de injúria, sendo que ambos influenciam na depleção muscular do indivíduo, principalmente em características fisiológica e psicológica, dentre elas à diminuição do apetite por dor ou receio, palatabilidade e aceitabilidade da dieta, uma vez que o mal prognóstico da doença se torna fator desencadeante para predisposição à infecções e à diminuição da força dos músculos expiratórios afetando diretamente do processo de catabolismo na recuperação e qualidade de vida do paciente (BARROS, et al., 2003). Portanto, sugere-se uma investigação em relação ao diagnóstico clínico dos pacientes avaliados, para melhor ponderação das classificações de estado nutricional, principalmente magreza e eutrofia que se destacam nos resultados.

Quanto à aplicação do IMC para o processo de triagem dos pacientes avaliados observa-se em outros estudos que há uma incompatibilidade de eficiência para tal diagnóstico de avaliação mais profunda, porém para a finalidade de triar aqueles que apresentam riscos nutricional generalizado, esta se torna funcional em termos de agilidade. Nesse sentido, em um estudo científico, Ricardo e Araújo (2002), com objetividade, realizaram uma crítica à utilização do IMC como ferramenta avaliativa do estado nutricional de indivíduos, comparando-a à aplicação de duas metodologias, sendo elas: índice ponderal e ectomografia. Concluindo assim, que a determinação do IMC, não reflete a composição corporal e apresenta limitações matemáticas intrínsecas como método, principalmente nos extremos dos dados de altura.

\section{CONCLUSÃO}

Com base nos resultados deste estudo, de acordo com a classificação do IMC, verificou-se que $7 \%$ dos pacientes avaliados encontrava-se em estado nutricional de magreza. No entanto, mediante a utilização isolada desta ferramenta avaliativa, não foi possível determinar se o restante dos pacientes triados apresentava outras deficiências em relação à composição corporal.

Dessa maneira, infere-se que a classificação do estado nutricional mediante o IMC calculado com os dados coletados de pacientes hospitalizados, não é determinante para estabelecer um possível risco nutricional dos mesmos, pois utilizase somente dados de peso e altura. Dessa maneira, este parâmetro deveria ser conjugado a outras informações que considerem outras questões sobre a composição corporal do paciente, tais como pregas cutâneas, para assim, determinar com exatidão possíveis perdas de massa magra, que configura um risco nutricional. 


\section{REFERÊNCIAS}

BARROS, S.E; MOURA, L.S.A.; CARVALHO, M.J.C.; OLIVEIRA, MOREIRA, A.; OLIVEIRA, C.R. Correlação das Pressões Respiratórias Máximas com o Estado Nutricional de Doentes Pulmonares. Rev Bras Nutr Clin 2003; 18 (3).

BOSCO, V.E..; SOUZA, K.M.M.; VIANA, K.D.À.L.; CALADO, I.L. Estado nutricional de indivíduos hospitalizados em um hospital universitário de São Luís, Maranhão. Rev Pesq Saúde 2013.

BRASIL. Portaria 272 MS/SVS de 08 de abril de 1998. Regulamento Técnico para fixar os requisitos mínimos exigidos para a Terapia de Nutrição Parenteral. Secretária de Vigilância Sanitária do Ministério da Saúde, 1998.

CHUMLEA, W.M.C.; GUO, S.S.; ROCHE, A.F.; STEINBAUGH, M.L. Prediction of body weight for the nonambulatory elderly from anthropometry. J Am Diet Assoc 88(5):564-8, 1985.

DIAS, M.C.G.; HORIE, L.M.; WAITZBERG, D.L. Nutrição oral, enteral e parenteral na prática clínica. $4^{\underline{a}}$ ed. São Paulo: Ed. Atheneu: 2009.

DUCHINI, L.; JORDÃO, A.A.; BRITO, T.T.; DIEZ-GARCIA, R.W. Avaliação e monitoramento do estado nutricional de pacientes hospitalizados: uma proposta apoiada na opinião da comunidade científica. Rev. Nutr., Campinas, 23(4):513-522, 2010.

FERNANDES, À.C.; BEZERRA, O.M.P.A. Terapia nutricional na doença pulmonar obstrutiva crônica e suas complicações nutricionais. J. bras. pneumol. vol.32 no.5 São Paulo Sept./Oct. 2006.

FIDELIX, M.S.P.; SANTANA, A.F.F.; GOMES, J.R. Prevalência de desnutrição hospitalar em idosos. Revista da Associação Brasileira de Nutrição-RASBRAN, 5(1):60-68, 2013.

FRANCO, J.B.; MORSOLETTO, R. H. C. Perfil nutricional de pacientes hospitalizados em um hospital público do município de Ituiutaba, Minas Gerais. Rev Bras Nutr Clin; 27 (3): 187-92, 2012.

MAHAN, L.K.; RAYMOND, J.L. Krause Alimentos, Nutrição e Dietoterapia. $14^{\circ}$ Edição. São Paulo, SP: Elsevier, 2018.

MITCHELL, C.O.; LIPSCHITZ, D.A. Arm length measurement as an alternative to height in nutritional assessment of the elderly. J Parenter Enteral Nutr, v.6, n.3, p.226-9, 1982.

PAULA, J.À.; WAMSER, E.L.; GOMES, À.R.S.; VALDERRAMAS, S.R.; NETO, J.C; SCHIEFERDECKER, M.E.M. Análise de métodos para detectar sarcopenia em idosas independentes da comunidade. Universidade Federal do Paraná, Curitiba PR, Rev. Bras. Geriatr. Gerontol., Rio de Janeiro, 2016. 
RICARDO, D.R.; ARAÚJO, C.G.S. Índice de Massa Corporal: Um Questionamento Científico Baseado em Evidência. Arq Bras Cardiol, v.79, n.1, p.61-9, 2002. 